\title{
AETIOLOGY AND OUTCOME OF ACUTE KIDNEY FAILURE IN BANGLADESHI CHILDREN - DHAKA MEDICAL COLLEGE HOSPITAL EXPERIENCE
}

\author{
AFROZ $\mathrm{S}^{1}, \mathrm{SIMI} \mathrm{MA}^{2}, \mathrm{SHARMIM} \mathrm{S}^{3}, \mathrm{KHANUM} \mathrm{R}^{4}, \mathrm{YEASMIN} \mathrm{L}^{5}, \mathrm{KUNDO}^{2}{ }^{6}$, AHMED AS $^{7}$, \\ RAHMAN F ${ }^{8}$
}

\begin{abstract}
Background: Acute Kidney Failure (AKF) is the acute loss of kidney function over hours or days, the aetiology of which varies in different countries.

Objectives: The study was aimed to find out the aetiology and see the immediate outcome of $A K F$ in children in a tertiary level teaching hospital in Bangladesh.

Methods: This prospective observational study was conducted from June 2011 to December 2012 in the Paediatric Nephrology Department of Dhaka Medical College Hospital, Dhaka. AKF was evidenced by presence of any two: serum creatinine raised $>3$ fold from the baseline, urine output $<0.3 \mathrm{ml} / \mathrm{kg} / \mathrm{hr}$ for 24 hours or anuria for 12 hours. A total of 50 children with AKF were evaluated regarding their socio-demographic and clinical data. All underwent intermittent peritoneal dialysis and other necessary supportive care as needed. They were followed up for 3 months after enrolment.
\end{abstract}

Results: Age of the children ranged from 5 days to 12 years. Among them, 33 were male and 17 female. Majority of the patient were from rural area and from a poor socioeconomic status. Out of 50 children majority (76\%) were under 5 year age. The aetiology of AKF was mainly prerenal 66\%, followed by renal $32 \%$, post renal 2\%children. Among the pre-renal AKF 68\% were due to diarrheal complications, $12 \%$ neonatal AKF were due to severe perinatal asphyxia (PNA). Haemolytic uraemic syndrome, wasp envenomation and acute glomerulonephritis were the common renal causes of $A K F$. Post-renal cause of $A K F$ was due to posterior urethral valve. The overall 38(76\%) survived with normal renal functions, 10(20\%) died and 2(4\%) developed chronic kidney disease. The higher mortality rate was due to late referral, multi organ failure and severe degree of renal failure.

Conclusion: This study suggests that diarrhoeal disease was the major cause of paediatric $A K F . A K F$ is more common in infancy and associated with increased mortality. Common cause of neonatal $A K F$ is severe perinatal asphyxia.

Key word: Aetiology, acute kidney failure (AKF), chronic kidney disease (CKD).

J Dhaka Med Coll. 2015; 24(2) : 86-91.

\section{Introduction}

Acute kidney failure $(\mathrm{AKF})$ in children is a catastrophic event. Acute kidney injury is characterized by a reversible increase in the blood concentration of creatinine and nitrogenous waste products and by the inability of the kidney to regulate fluid and electrolyte homeostasis appropriately. The incidence of AKF in children appears to be increasing, and the aetiology of AKF over the past decades has

1. Dr. Shireen Afroz, Associate Professor, Dept. of Paediatric Nephrology, Dhaka Medical College Hospital, Dhaka.

2. Dr .Maliha Alam Simi, Registrar, Dept. of Paediatrics, Dhaka Medical College Hospital, Dhaka.

3. Dr. Shanjida Sharmim, Assistant Registrar, Dept. of Paediatrics, Dhaka Medical College Hospital, Dhaka.

4. Dr. Rokeya Khanum, Assistant Professor, Dept. of Paediatrics, Dhaka Medical College Hospital, Dhaka.

5. Dr. Laila Yeasmin, Assistant Professor, Dept. of Paediatrics, Dhaka Medical College Hospital, Dhaka.

6. Dr. L.C. Kundo, Assistant Professor, Dept. of Paediatrics, Faridpur Medical College Hospital, Faridpur.

7. Dr. Abu Saleh Ahmed, Associate Professor, Dept. of Nephrology, Kustia Medical College, Kustia

8. Dr. Farzana Rahman, Research Assistant, Dept. of Paediatrics, Dhaka Medical College Hospital, Dhaka.

Correspondence: Dr. Shireen Afroz, Associate Professor, Dept. of Paediatric Nephrology, Dhaka Medical College Hospital, Dhaka. Cell Phone: +8801715054239, Email: safroz16@live.com 
shifted from primary renal disease to multifactorial causes, particularly in hospitalized children ${ }^{1}$. Renal injury can be divided into prerenal disease, renal disease including vascular insults, and post renal disease. The prognosis of AKF is highly dependent on the underlying aetiology of the AKF. Children who suffered AKF from any cause are at risk of developing chronic kidney disease several years after the initial insult ${ }^{2}$. The available literature suggests that not only the severity of AKF (needing dialysis), but also the mild forms of AKF may have an impact on morbidity and mortality of affected patients ${ }^{3}$. Furthermore, identifying patients in the early stage of $\mathrm{AKF}$ and identifying the risk factors associated with those early stages may help in preventing fur-ther worsening of renal function if timely inter-vention is undertaken ${ }^{4}$. Paracetamol elixirs with diethylene glycol as diluent was held responsible for a large outbreak of fatal renal failure in Bangladesh ${ }^{5}$. Besides, there is many other cause of AKI in Bangladesh. This study was done to find out the aetiology and immediate outcome of acute kidney failure in children who received intermittent peritoneal dialysis in Dhaka Medical College Hospital, Dhaka. Thus improved understanding of the incidence, aetiology and outcomes of paediatric AKF will help in designing effective therapeutic strategies for early diagnosis and intervention of AKF in children.

\section{Methods}

This prospective observational descriptive study was carried out at Paediatric Nephrology Department of Dhaka Medical College Hospital, Dhaka, conducted over 19 months, from June 2011 to December 2012. Hospitalized children between 5 days and 12 years were included in this study. Total population was divided into four age groups: neonate, 1 month to 12 months, 13 months to 5 years and 6 to 12 years. Objectives of the study were to determine the aetiology and immediate outcomes (within 3 month) of AKF in children hospitalized at a tertiary level who received intermittent peritoneal dialysis (IPD). All children with AKF within above mentioned period were evaluated on their socio-demographic, clinical and biochemical aspect. Pre-renal, renal and postrenal causes of AKF were explored.

AKF was considered when sudden deterioration of kidney function evidenced by any two: serum creatinine raised $>3$ fold from the baseline, urine output $<0.3 \mathrm{ml} / \mathrm{kg} / \mathrm{hr}$ for 24 hours or anuria for 12 hours. Base line serum creatinine was estimated by using the levels of published serum creatinine value of normal children according to age ${ }^{6}$. To be considered acute, renal failure had to develop over less than 72 hours. Hypotension was defined as sys-tolic blood pressure less than $70 \mathrm{~mm} \mathrm{Hg}+$ $2 \times$ age in years. Hyperkalaemia was defined as se-rum potassium of $<6.5 \mathrm{meq} / \mathrm{L}$. Hyponatremia was considered when serum sodium is <125 meq/L. Multi-organ failure(MOF) was defined as dysfunction of more than two vital organs. Late referral when admitted following 48 hours after clinical complaints, anuria for 2 day or more was also noted as severity of AKF.

Exclusion criteria were age $>12$ year, AKF on Chronic Kidney Disease (CKD), AKF with other systemic diseases, malignancy. Prior to the commencement of the study parents were adequately counselled about the nature of the study and written informed consent was also taken.

\section{Results}

A total of 50 children with AKF were included in this study. Age of the children ranged from 5 days to 12 years. Among them 4(8\%) were neonate, $15(30 \%)$ were in 1 month to 12 months age group, $16(32 \%)$ in 13 months to 5 years age group, $15(30 \%)$ in 6 to 12 years age group (fig. 1). Majority were under 5 children (76\%). (Fig. 1); and 33 were male and 17 were female. Male to female ratio was 1.9: 1. Majority of the patient were from rural area (64\%) and from a poor $(32 \%)$ socioeconomic status. About 58\% mothers were illiterate (Table I). The study revealed aetiology of AKF was mainly pre renal in $66 \%(n=33)$, followed by renal $32 \%(n=16)$, post-renal $2 \%(n=1)$ children (Fig. 2). Among the 
pre-renal AKF (table-II), 82\% (27) were due to diarrhoeal complications, $12 \%(n=4)$ neonatal AKF were due to severe perinatal asphyxia (PNA). Renal causes include haemolytic uraemic syndrome $\mathrm{n}=7(43 \%)$, wasp envenomation $\mathrm{n}=3(18 \%)$ and post-streptococcal acute glomerulonephritis $n=2(12 \%)$ were the common renal causes of AKF (Table II).

\section{Table I}

Socio demographic data

\section{Socioeconomic status}

Poor

$16(32 \%)$

Lower middle class

$29(58 \%)$

Middle class

$5(10 \%)$

\section{Residence}

Urban

$18(36 \%)$

Rural $32(64 \%)$

\section{Maternal educational status}

Illiterate

$29(58 \%)$

Literate $21(42 \%)$

Post-renal cause of AKF was due to posterior urethral valve $n=1(2 \%)$. The overall $76 \%(n=38)$ survived with normal renal functions, $20 \%$ $(n=10)$ died and $4 \%(n=2)$ developed chronic kidney disease ( 1 in CKD stage II and another in CKD stage III) evidenced by persistent rise of serum creatinine during follow up after 3

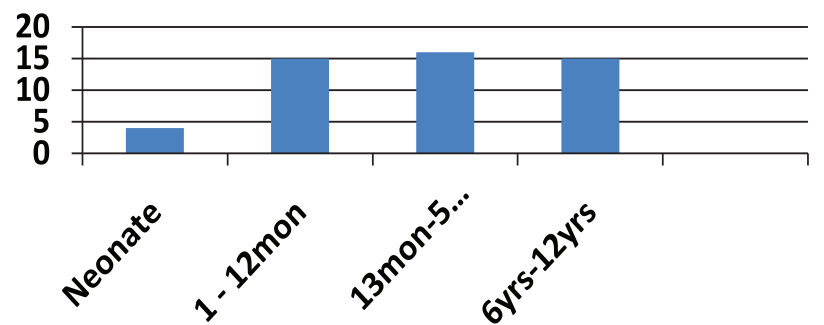

Fig.-1: Age distribution of the study children $(n=50)$

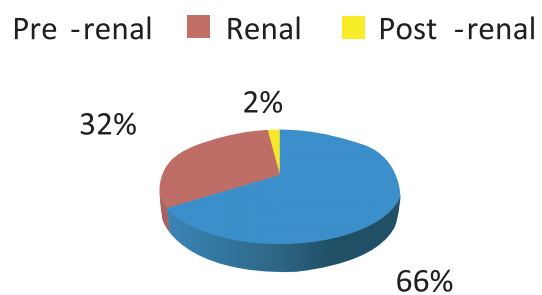

Fig.-2: Causes of AKF according to pre-renal, renal \& post-renal involvement

months. Cause of death in neonate was multiorgan involvement. Most perinatal asphyxia neonate developed sepsis, shock, jaundice and dyselectrolytaemia. Mortality was high in neonate $(50 \%)$ and under 5 children (26\%). Outcome was excellent with normal renal functions in children older than 5 years (Table III). Cause of death in infants and under 5 children were late referral, prolong duration of anuria, with severe degrees of dehydration, acidosis, dyselectrolytaemia and MOF (Table IV).

Table-II

Distribution of causes of AKF according to diseases

\begin{tabular}{lcc}
\hline Pre-renal causes $(\mathrm{N}=33)$ & Renal causes $(\mathrm{N}=16)$ & Post renal causes $(\mathrm{N}=1)$ \\
\hline Post-diarrhoeal AKF due to & HUS $7(43.6 \%)$ & Posterior urethral value \\
hypovolemia/ shock $27(82 \%)$ & & \\
Perinatal asphyxia 4(12\%) & Wasp 3(18.8\%) & \\
Post-surgical 2(6\%) & AGN 2(12.5\%) & \\
& RPGN 2(12.5\%) & \\
& Drugs $2(12.5 \%)$ & \\
\hline
\end{tabular}




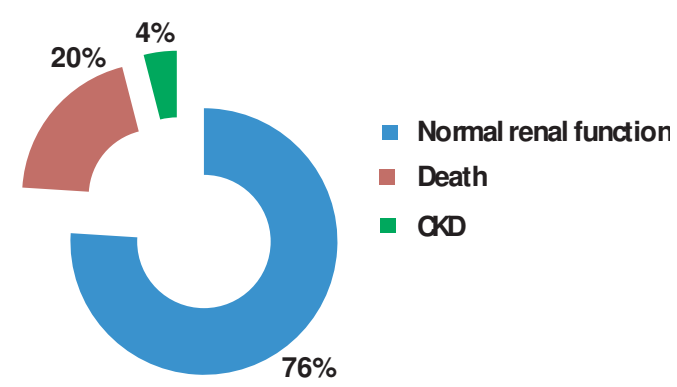

Fig.-3: Outcome of the study children with $A K F$ $(N=50)$

Table-III

Outcome according to age distribution

\begin{tabular}{lccc}
\hline Age distribution & No (\%) & Survived & Death \\
\hline Neonate & $4(8)$ & 2 & 2 \\
$>1$ month - & $15(30)$ & 11 & 4 \\
12 months & & & \\
13months - 5yr & $16(32)$ & 12 & 4 \\
$>$ 5yr - 12yr & $15(30)$ & 15 & 0 \\
\hline Total & 50 & 40 & 10 \\
\hline
\end{tabular}

Table-IV

Cause of death in study children of $A K F N=10$

\begin{tabular}{lcc}
\hline & $\begin{array}{c}\text { Neonate } \\
\mathrm{n}=2\end{array}$ & $\begin{array}{c}\text { Under 5 } \\
\text { children } \\
\mathrm{n}=8\end{array}$ \\
\hline Sepsis & 2 & 4 \\
Shock/ \& severe dehydration & 1 & 6 \\
Jaundice & 1 & 0 \\
Hyponatremia & 2 & 5 \\
Late referral 48 hours after & 1 & 4 \\
clinical complaints & & \\
Anuria for 2 day or more & 0 & 6 \\
Pneumonia & 0 & 2 \\
Congestive heartfailure & 0 & 1 \\
MOF & 2 & 5 \\
\hline
\end{tabular}

\section{Discussion}

AKF is a life-threatening condition, especially in children, with significantly increased morbidity and mortality rates ${ }^{7}$. As Dhaka Medical College Hospital is one of the major tertiary care centres in Bangladesh, many of the severe or complicated cases from different regions of the country are referred here. Most of the patients are poor and some belong to lower middle class family. So, they get treatment here at free of cost.In this study we have evaluated these complicated AKF who were treated with IPD to explore the aetiology and outcome. In the present study AKF was mainly pre-renal (66\%). Among the pre-renal AKF, 68\% were due to diarrheal complications, $12 \%$ neonatal AKF were due to severe perinatal asphyxia (PNA). Hypovolemia was the most common cause of AKF in our study. The cause of hypovolemia was mostly due to diarrhoea. We got a few neonates who presented with $\mathrm{AKF}$ as a complication of perinatal asphyxia. Haemolytic uraemic syndrome, wasp envenomation and acute glomerulonephritis were the other causes of AKF. Post-renal cause of AKF was due to posterior urethral valve. Rahman et al. ${ }^{8}$ also found higher number of hypovolemic AKF in children in a study at IPGMR in Bangladesh and concluded that acute ischemic renal failure in children bears a much poorer prognosis compared to that of AKF due to HUS.

Haemolytic uraemic syndrome was reported as a major cause of AKF in some studies too ${ }^{9,10}$. Bailey et al reported that more than $50 \%$ of $\mathrm{AKF}$ cases were due to HUS, haematological malignancies or car-diac surgeries ${ }^{11}$. The aetiology of AKF seems to differ in diffe-rent countries $^{12}$. In Kuwait, sepsis was found to be the most common cause of AKF accounting for $46.9 \%$ of cases followed by haematological malignancies complicated with the tumourlysis syndrome ${ }^{13}$. Flynn et al. reported postoperative sepsis as a prominent cause of $\mathrm{AKF}$ in the paediatric population ${ }^{14}$. Several other studies also have reported sepsis as the major cause of AKF in children $7,13,15,16$. However, in developed countries cardiovascular surgeries and the haemolytic uremic syndrome (HUS) are the most important risk factors associated with AKF in the paediatric population ${ }^{9,10}$. On the other hand, Vachvavichsanong et al. reported that haematological malignancies involving the kidneys and neph-rotoxic chemotherapy were less common causes; however, children with malignancies tend to develop sepsis particularly during 
chemo-therapy ${ }^{7}$. The overall survival rate in this study is $76 \%$ with normal renal functions, $20 \%$ died and $4 \%$ developed chronic kidney disease. Mortality rates in children with AKF tend to be high $43.8 \%$ in a study done in Kuwait ${ }^{13}$. Mortality rates of $25--50 \%$ have been reported in other studies depen-ding on the underlying disorder ${ }^{17-22}$.

Our study showed that the mortality rate is higher in younger children. The same was re-ported by Vachvanichsanonget al. ${ }^{7}$.Several studies have reported that the cause of AKF was significantly associated with age, but, age by itself was not an independent predictor of mortality $7,21,23$. Children with MOF were found to have a higher mortality $(88.3 \%)^{12}$. Pre-vious studies have documented that when the kidneys are involved in MOF, the prognosis is very poor with a mortality rate of $45-75 \%$. In contrast, the prognosis is better when primary renal disorder is the cause of $\mathrm{AKF}$, wherein the mortality rate is 9 to $12.5 \% 11,17,19$. The outcome of renal replacement therapy (RRT) in children varies throughout the world ${ }^{11}$. The present study showed favourable outcome following peritoneal dialysis which is safe and easier for young children. Otukesh et al. showed a higher mortality rate in patients treated with haemo-dialysis than those treated with peritoneal dialysis and attributed this to the recurrent hypotensive episodes that occur during haemo-dialysis ${ }^{11}$.

\section{Conclusion}

Pre-renal AKF especially diarrhoeal disease is the commonest cause of AKF found in this study. AKF is more common in infancy and under 5 children and associated with increased mortality rate. Common cause of neonatal AKF is perinatal asphyxia with worst prognosis. The higher mortality rate was due to late referral, multi-organ failure and severe degree of renal failure.

\section{References}

1. Andreoli SP. Acute kidney injury in children. Pediatr Nephrol 2009; 24(2): 253-63.

2. Yan Cao, Zhu-Wen Yi, Hui Zhang, et al. Etiology and outcomes of acute kidney injuries in Chinese children: a prospective multicentre investigation. BMC Urology 2013; 13:41.
3. Ronco C, Bellomo R, Homel P, et al. Effects of different doses in continuous venovenous hemo-filtration on outcome of acute renal failure: a prospective randomized trial. Lancet 2000; 356(9223):26-30.

4. Lowrie L. Renal replacement therapies in pediatric multi-organ dysfunction syndrome. Pediatr Nephrol 2000;14(1): 6-12.

5. Hanif M, Mobarak MR, Ronan A, et al. Fatal renal failure caused by diethylene glycol in paracetamol elixir: the Bangladesh epidemic. BMJ 1995; 311(6997):88-91.

6. Ceriotti F, Boyd JC, Klein G, et al. Reference intervals for serum creatinine concentrations: assessment of available data for global application. ClinChem 2008;54(3):559-66.

7. Vachvanichsanong $\mathrm{P}$, Dissaneewate $\mathrm{P}, \mathrm{Lim} \mathrm{A}$, et al. Childhood acute renal failure: 22-years experience in the university hospital in southern Thailand. Pediatrics 2006;18(3):786-91.

8. Rahman $\mathbf{M}^{1}$, Hossain $\mathrm{M}$. The bimodal mortality pattern of acute renal failure in children. Ren Fail 1993;15(1):55-9.

9. Spizzirri FD, Rahman RC, Bibiloni N, Ruscasso JD, Amoreo OR. Childhood hemolytic uremic syndrome in Argentina: Long term follow-up and prognostic features. Pediatr Nephrol 1997; $11(2): 156-60$.

10. Moghal NE, Brocklebank JT, Meadow SR. A review of acute renal failure in children: incidence, etiology and outcome. Clin Nephrol 1998;49:91-5.

11. Bailey D, Phan V, Litalien C, et al. Risk factors of acute renal failure in critically ill children: a retrospective epidemiological study. Pediatr Crit Care Med 2007;8(1):29-35.

12. Otukesk H, Hoseini R, Hooman N, et al. Prognosis of acute renal failure in children. Pediatr Nephrol 2006; 21(12):1873-8.

13. Ghani AA, Al Helal B, Hussain N. Acute renal failure in pediatric patients: etiology and predictors of outcome. Saudi J Kidney Dis Transpl 2009;20:69-76.

14. Flynn JT. Causes, management approaches and outcome of acute renal failure in children. CurrOpin Pediatr.1998;10:184-9.

15. Smoyer WE, McAdams C, Kaplan BS, et al. Determinants of survival in pediatric continuous hemofiltration. J Am SocNephrol1995;6(5): 1401-9.

16. Loza R, Estremadoyro L, Loza C, et al. Factors associated with mortality in acute renal failure 
in children. Pediatr Nephrol 2006; 21(1): 106-9.

17. Gallego N, Perez-Caballero C, Gallego A, et al. Prognosis of patients with acute renal failure without cardio-pathy. Arch Dis Child 2001;84(3):258-60.

18. Fitzpatrick MM, Kerr SA, Bradbury MG. Acute renal failure. In: Webb N, Post-lethwaite R, eds. Clinical Pediatric Nephrology. 3rd ed. Oxford: Oxford University Press; 2003:405-25.

19. Arora P, Kher V, Rai PK, et al. Prognosis of acute renal failure in children: a multivariate analysis. Pediatr Nephrol 1997;11(2):153-5.
20. Anochei IC, Eke FU. Acute renal failure in Nigerian children: Port Harcourt experience. Pediatr Nephrol 2005;20(11):1610-4.

21. Hui-Stickle S, Brewer ED, Goldstein SL. Pedia-tric acute renal failure epidemiology at a tertiary care center from 1999 to 2001. Am J Kidney Dis 2005; 45:96-101.

22. Williams DM, Sreedhar SS, Mickell JJ, et al. Acute kidney failure: a pediatric experience over 20 years. Arch Pediatr Adolesc Med 2002; 156(9):893-900.

23. Liano F, Pascual J. Epidemiology of acute renal failure: a prospective multicenter, community based study, Madrid acute renal failure study group. Kidney Int 1996; 50(3): 811-8. 